\title{
Variación de las tensiones residuales con la deformación plástica en el ensayo de tracción
}

\author{
J.A. Benito*, J. Jorba* y A. Roca**
}

\begin{abstract}
Resumen Se ha estudiado la variación de las tensiones residuales longitudinales y transversales, mediante difracción de Rayos X, a medida que se aumenta el porcentaje de deformación plástica, medida como alargamiento porcentual (A \%), en un ensayo de tracción convencional. El material original, hierro Armco laminado en caliente, tiene valores de tensión residual cercanos a cero en la dirección longitudinal y alcanza valores de hasta 600 MPa a partir de $\mathrm{A}=1,5 \%$, manteniéndose este valor constante hasta la aparición de la estricción. En la dirección transversal los valores iniciales son de $-300 \mathrm{MPa}$, las variaciones son menores y la tensión residual permanece siempre compresiva. Se presenta además un estudio de la variación de la tensión residual con el tiempo y con el diferente grado de desalineamiento entre el rayo incidente y la dirección de estirado.
\end{abstract}

Palabras clave Tensiones residuales. Hierro puro. Trabajo en frío. Estirado.

\section{Change of residual stresses during plastic deformation under uniaxial tension test}

\begin{abstract}
hange of longitudinal and transverse residual stresses was studied by X Ray diffraction method as the applied plastic deformation, measured as A \%, was increased in a standard tension test. The starting material, hot rolling Armco iron, has values close to $0 \mathrm{MPa}$ in longitudinal direction, but it reaches $600 \mathrm{MPa}$ with only $\mathrm{A}=1,5 \%$, this value remains constant until necking is produced. In transverse direction the starting values are -300 $\mathrm{MPa}$, changes are small and residual stresses remain compressive until the end of tension test. In addition, studies of the changes of residual stresses with time and with misalignment between incident X Ray and drawing direction are presented.
\end{abstract}

Keywords

Residual stresses. Pure iron. Cold working. Drawing.

\section{INTRODUCCIÓN}

Existen muchas situaciones donde es necesario conocer el estado de las tensiones residuales de un material puesto que su efecto, perjudicial o beneficioso, puede modificar su respuesta. Por ello, se han desarrollado métodos no destructivos para la determinación de tensiones residuales ${ }^{[1]}$. Dentro de los materiales metálicos, la deformación en frío provoca variaciones en estas tensiones, que son diferentes dependiendo de la cantidad y del tipo de deformación utilizado.

En el caso concreto del ensayo de tracción, se han realizado estudios ${ }^{[2]}$ para conocer cómo afecta este ensayo a las tensiones residuales introducidas previamente en la superficie del material mediante tratamientos de granallado (shot peening). En estos estudios se observa cómo la deformación plástica que sufre el material durante el ensayo de tracción provoca la desaparición de las tensiones residuales compresivas en la superficie.

Dentro del estudio de la variación de las constantes elásticas de diferentes metales con la deformación en frío, es importante conocer si la diferente respuesta elástica (módulo de elasticidad) que se observa ${ }^{[3]}$, a diferentes estados de deformación, puede estar provocada por diferentes estados de tensiones residuales.

El objetivo de este trabajo es reproducir el comportamiento de las tensiones residuales a medida

(*) Unidad de Mecánica Aplicada. EUETI Barcelona-UPC. Comte d'Urgell 187.08036 Barcelona (España).

(**) Dept. de Ingeniería Química i Metalurgia. Facultad de Química. Universidad de Barcelona. Martí y Franqués, 1. 08028 Barcelona (España). 
que aumenta la deformación plástica medida como alargamiento porcentual (A \%) y realizada mediante un ensayo de tracción, sobre un material que no ha sufrido un tratamiento previo. En este caso, nuestro material es un hierro que se encuentra en un estado de deformación en caliente, por lo que se parte de unos niveles de tensiones residuales prácticamente nulos. Las mediciones se realizan antes y después de cada estado de deformación plástica, hasta la aparición de la estricción.

\section{MÉTODO EXPERIMENTAL}

Se utilizó como material de partida lámina de hierro Armco laminado en caliente. La composición del hierro Armco, según especificaciones del proveedor ${ }^{[4]}$ y de los análisis realizados posteriormente, se muestra en la tabla I. De este material se mecanizaron probetas de sección rectangular según la norma UNE 7-474-92 (EN 10002-1).

El método de trabajo se expone en la figura 1. El ensayo de tracción se interrumpe al alcanzarse un valor de alargamiento porcentual determinado

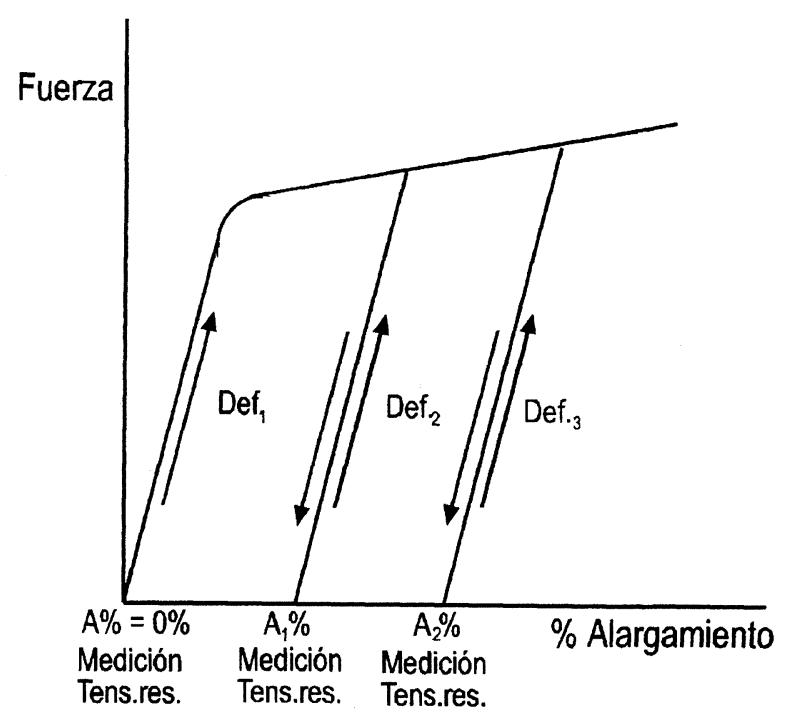

Figura 1. Procedimiento experimental. Después de cada deformación plástica se realiza la determinación de tensiones residuales.

Figure 1. Experimental procedure. Residual Stresses are determined affer each deformation process.
(A \%). Se realiza la determinación de tensiones residuales y la probeta se vuelve a deformar a otro valor de $\mathrm{A} \%$. El proceso se repite hasta la aparición de la estricción. El ensayo se llevó a cabo en una máquina de ensayos universal Instron 8501.

La determinación de la tensión residual en la superficie de cada probeta se realizó mediante difracción de Rayos X por el método de $\sin ^{2} \Psi^{[5]}$ en un aparato Philips provisto de goniómetro de Schulz (método de reflexión). Se utilizó el pico de difracción $\{110\}$ para todas las medidas. Todas las medidas se realizaron dirigiendo el haz de Rayos X hacia el centro de la probeta. La desviación máxima en los valores calculados es de $\pm 30 \mathrm{MPa}$.

Para la determinación de las tensiones residuales longitudinales, la dirección de estirado y la dirección de emisión de los rayos X coinciden. Para las tensiones residuales en la dirección transversal, ambas son perpendiculares. Este ángulo entre las direcciones de estirado y emisión de rayos $\mathrm{X}$ se define como ángulo de desalineamiento, $\varphi$ (Fig.2), $0^{\circ}$ - para longitudinal y $90^{\circ}$ para transversal.

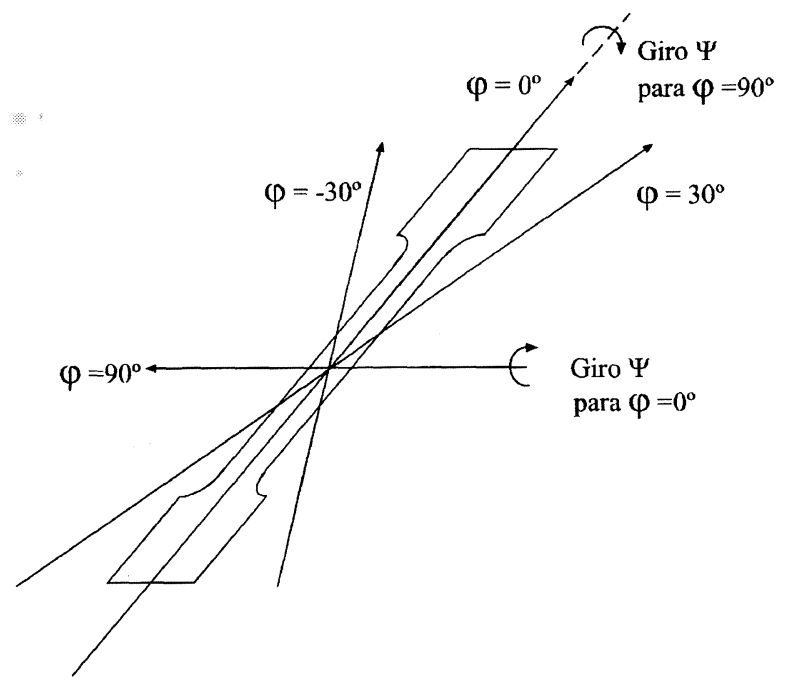

Figura 2. Disposición de los ángulos de desalineamiento $\varphi$ en las probetas de tracción y sentido de giro del ángulo $\Psi$ para las direcciones longitudinal y transversal.

Figure 2. Arrangement of misalignment angle $\varphi$ in tensile samples. It is shown how $\Psi$ angles rotates for longitudinal and transverse cases.

Tabla I. Composición química del hierro Armco (\% peso)

Table I. Chemical composition of Armco Iron (\% wt.)

\begin{tabular}{lcccccccc}
\hline & C & S & P & Si & Mn & Cu & Sn \\
\hline Especificaciones (máximo) & 0,010 & 0,005 & 0,010 & - & 0,08 & 0,03 & 0,010 \\
Análisis & 0,002 & 0,001 & 0,010 & 0,01 & 0,06 & 0,01 & 0,003 \\
\hline
\end{tabular}


El método $\sin ^{2} \Psi$ requiere el giro de la probeta a determinados valores del ángulo $\Psi$, según distintos ejes para cada dirección de medida (Fig 2). Debido a que había que probar la misma probeta a diferentes valores de alargamiento porcentual (A \%), se necesitaba la probeta entera, incluyendo los cabezales.

A la hora de determinar las tensiones residuales longitudinales, las probetas resultaron ser demasiado grandes para permitir el giro sobre $\psi$ para el ángulo $\varphi=0^{\circ}$, es decir, la dirección longitudinal. Para resolver el problema, se realizaron determinaciones de tensiones residuales de una probeta sin deformar $(\mathrm{A} \%=0 \%)$ y otra deformada $(\mathrm{A} \%=$ $11 \%$ ), a las que se habían eliminado los cabezales. Se realizaron diferentes mediciones variando el grado de desalineamiento (ángulo $\varphi$ ) entre el haz incidente y la dirección de estirado. Del estudio de los valores obtenidos (Fig. 3), se observa como hay poca diferencia para ángulos cercanos a $\varphi=0^{\circ}$, por lo que se decidió hacer las medidas de la dirección longitudinal con un ángulo $\varphi=30^{\circ}$, ya que con ese ángulo sí podían girar sobre $\psi$ las probetas enteras. Las tensiones residuales transversales se determinaron normalmente tomando un ángulo $\varphi=90^{\circ}$.

Con las dos probetas iniciales se comprobó, también que, en ambos casos, no había una variación significativa entre los valores de tensión entre una cara y la otra.

En todas las medidas, para determinar la existencia de tensiones de cizalla perpendiculares a la superficie, se realizaron medidas en la misma dirección, en ambos sentidos, con $\varphi=30^{\circ}$ y $210^{\circ}$ para longitudinal y $\varphi=90^{\circ}$ y $270^{\circ}$ para transversal.

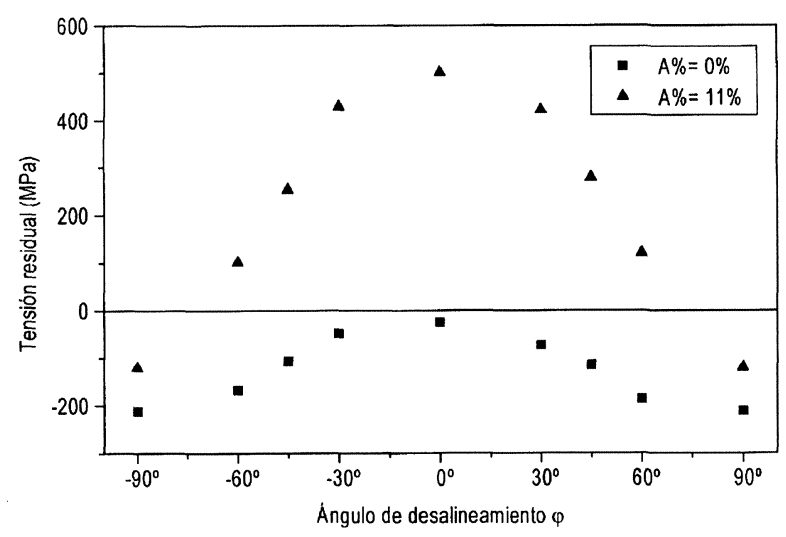

Figura 3. Variación de la tensión residual con el ángulo de desalineamiento $\varphi$.

Figure 3. Change of residual stress with misalignment angle $\varphi$.
Cada probeta se deformó mediante un ensayo de tracción convencional en una máquina de ensayos universal Instron 8501, interrumpiendo el ensayo cuando la deformación plástica llegaba al 1,5 $\%, 3 \%, 4,5 \%, 6 \%, 7,5 \%$... hasta la aparición de la estricción. En cada estado de deformación se realizó una determinación de las tensiones residuales.

\section{RESULTADOS Y DISCUSIÓN}

\subsection{Variación de la tensión residual con la de- formación plástica}

La figura 4 muestra los valores de la tensión residual para las tres probetas ensayadas en la dirección longitudinal. La variación del ángulo de ataque del haz incidente $\left(\varphi=30^{\circ}\right.$ y $\left.210^{\circ}\right)$ no produce cambios significativos en ningún momento, lo que descarta un gradiente de tensiones. También, se observa como se pasa, en todos los casos, de tensiones casi nulas en el estado inicial (entre -37 y 16 $\mathrm{MPa}$ ), a valores entre 547-618 $\mathrm{MPa}$, con tan sólo una deformación de $\mathrm{A} \%=1,5 \%$, que están por encima de los valores de la resistencia máxima del material $\left(\sigma_{\mathrm{M}}=300 \mathrm{MPa}\right)$.

A medida que se producen deformaciones mayores, la tensión residual en las probetas $11 \mathrm{D}$ y 13 D no experimenta variaciones significativas a medida que va aumentando el alargamiento, permaneciendo más o menos constante. Tan sólo la probeta $9 \mathrm{D}$ muestra diferencias entre estados de deformación y en general un ligero descenso de la tensión para valores de alargamiento altos, llegando a bajar hasta los $300 \mathrm{MPa}$.

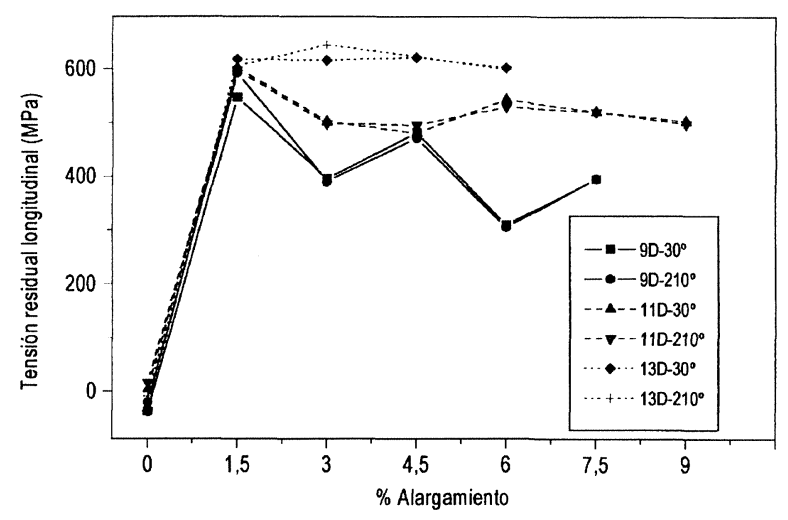

Figura 4. Variación de la tensión residual longitudinal con el alargamiento en las probetas 9D, 1 1D y 13D (medidas en $\varphi=30^{\circ}$ y $210^{\circ}$ ).

Figure 4. Change of longitudinal residual stress with elongation in samples 9D, 11D and 13D (measured for $\varphi=$ $30^{\circ}$ y $210^{\circ}$.

Rev. Metal. Madrid 37 (2001) 
En la figura 5 se presentan los valores de la tensión residual en la dirección transversal para dos de las tres probetas anteriores. En este caso, la tensión original es claramente compresiva (se sitúa entre -190 y $-294 \mathrm{MPa}$ ) y se observa un comportamiento menos acusado con la deformación, puesto que la tensión se relaja progresivamente, alcanzando valores relativamente constantes (entre -95 y $-135 \mathrm{MPa}$ ) a partir de un alargamiento del 6 $\%$.

\subsection{Relajación de la tensión residual con el tiempo}

Se realizaron pruebas para determinar la posible influencia que podía tener el tiempo transcurrido entre la deformación y la determinación de las tensiones residuales. En la figura 6 se puede observar como la tensión residual a tracción disminuye en la dirección longitudinal a medida que el tiempo es mayor, alcanzando un descenso del $10 \%$ una semana después. En la dirección transversal la tensión residual compresiva también disminuye. En ambos casos, la relajación de la tensión parece que se amortigua con el paso de los días, hasta llegar a un valor estable.

Conocido este efecto, las determinaciones de tensión residual se realizaron en las probetas $11 \mathrm{D}$ y $13 \mathrm{D}$ el mismo día que la deformación a tracción. El estudio en la probeta $9 \mathrm{D}$ se realizó con anterioridad sin tener en cuenta este efecto, por lo que el tiempo transcurrido hasta la medición de las tensiones residuales podía variar hasta una semana entre los diferentes estados de deformación. Este

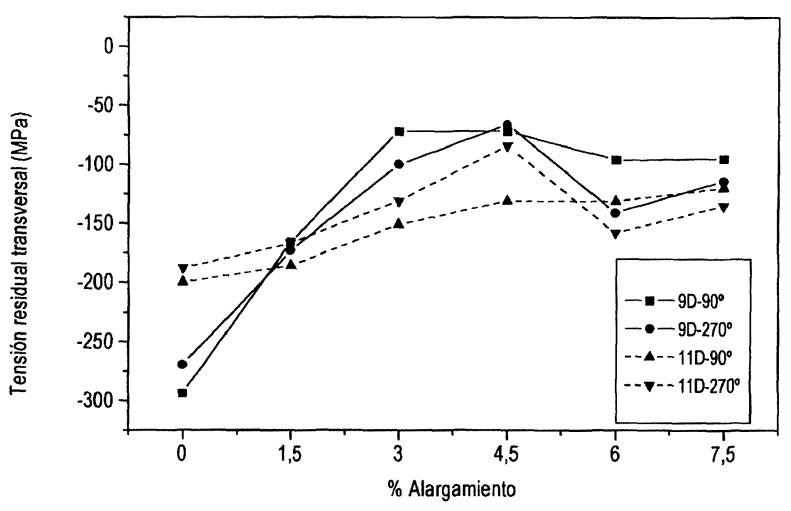

Figura 5. Variación de la tensión residual transversal con el alargamiento en las probetas 9D y 11D (medidas en $\varphi=90^{\circ}$ y $\left.270^{\circ}\right)$

Figure 5. Change of transverse residual stress with elongation in samples $9 D$ and $11 D$ (measured for $\varphi=90^{\circ} \mathrm{y}$ $270 \%$.

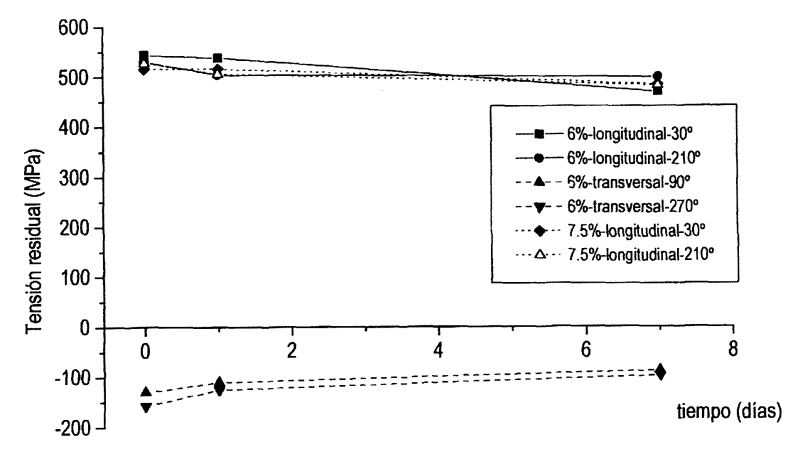

Figura 6. Variación de la tensión residual con el tiempo.

Figure 6. Change of residual stress with time.

hecho podría explicar las variaciones que sufre la tensión residual en esta probeta.

Los resultados del estudio muestran cómo, en la dirección longitudinal, el aumento de las tensiones residuales a tracción no es progresivo a medida que se incrementa la deformación sino que aparece bruscamente con A \% mínimos. La posterior deformación no incrementa la tensión sino que, en todo caso, la amortigua ligeramente. En la dirección transversal los cambios son pequeños y más progresivos.

A la hora de realizar mediciones de tensiones residuales después de una deformación conviene, de los resultados mostrados en la figura 6, realizarlas siempre contando con que existe una relajación de la tensión con el tiempo, que puede dar lugar a variaciones de un $10 \%$ en una semana.

\section{Agradecimientos}

Los autores quieren expresar su agradecimiento a Xavier Alcobé y Pep Bassas de la Unidad de difracción de Rayos X de los "Serveis Cientificotècnics" de la Universitat de Barcelona.

\section{REFERENCIAS}

[1] I.C. Noyan y J.B. Cohen, Am. Sci. 79 (1991) 142-153.

[2] T. Gurova, J.R. Teodosio, J. Rebello y V. Monin, Scr. Mater. 36 (1997) 1031-1035,.

[3] J.A. Benito, J. Calle, A. Roca y J. Jorba. V Congreso nacional de propiedades mecánicas de sólidos, ETSII Barcelona, UPC, 1996, pp.314-319.

[4] Armco Speciality Steels Europe. Armco Ingot Iron-pure Iron for remelting, Alemania, Nov.1994

[5] A.J. Perry, L. Chollet, J. Vacuum Sci. Technol. A 4 (1986) 2801-2807. 